\title{
EFECTO ANTIHIPERTENSIVO DEL EXTRACTO HIDROALCOHÓLICO DE LAS HOJAS DE Xanthium catharticum HBK “AMOR SECO”
}

\author{
Antihipertensive effect of the extract hidroalcohólic of Xanthium catharticum HBK "Amor seco" leaves \\ Zeyla Riveros ${ }^{1}$, Mario Carhuapoma ${ }^{2}$, Johnny A. Tinco ${ }^{1}$, Sofía López ${ }^{2}$, José Iannacone ${ }^{3}$ \\ ${ }^{1}$ Facultad de Ciencias de la Salud, Universidad Nacional de San Cristóbal de Huamanga. ${ }^{2}$ Facultad de Farmacia y Bioquímica, \\ Universidad Nacional Mayor de San Marcos. ${ }^{3}$ Universidad Nacional Federico Villarreal - Universidad Ricardo Palma
}

\section{RESUMEN}

El presente trabajo tiene por objetivo determinar el efecto antihipertensivo del extracto hidroalcohólico de las hojas de Xanthium catharticum HBK "amor seco" en ratas con hipertensión inducida. Se utilizaron muestras de Xanthium catharticum HBK colectadas en el centro poblado de Mollepata, región Ayacucho. Los metabolitos secundarios presentes en el extracto se determinaron utilizando pruebas especificas de coloración y precipitación; los resultados indicaron la presencia de sesquiterpenos, alcaloides, compuestos fenólicos, flavonoides, saponinas, aminoácidos libres y antocianidinas. Para determinar el efecto antihipertensivo se uso el método de inducción por administración crónica con L-NAME en 30 ratas, distribuidas aleatoriamente en seis grupos, el primero recibió agua destilada; el segundo, L-NAME $40 \mathrm{mg} / \mathrm{kg}$; el tercer, cuarto y quinto grupos recibieron L-NAME $40 \mathrm{mg} / \mathrm{kg}$ más extracto 100, 200, $400 \mathrm{mg} / \mathrm{kg}$, respectivamente; el sexto grupo recibió L-NAME $40 \mathrm{mg} / \mathrm{kg}$ más captopril $100 \mathrm{mg} / \mathrm{kg}$. La dosis con mayor porcentaje de eficacia antihipertensiva fue de 400 $\mathrm{mg} / \mathrm{kg}$ con $40,58 \%$, mientras que los grupos de 100 y $200 \mathrm{mg} / \mathrm{kg}, 33,96$ y $28,54 \%$, respectivamente, con un comportamiento medianamente antihipertensivo $(p<0,05)$, respecto al grupo control que recibió captopril $100 \mathrm{mg} / \mathrm{kg}$ presentó eficacia en el $99,4 \%$ de casos. Se concluye, que en condiciones experimentales, el extracto hidroalcohólico de las hojas de Xanthium catharticum HBK. "amor seco" evidencia tener moderado efecto antihipertensivo.

Palabras clave: Hipertensión, Xanthium catharticum HBK, L-NAME, amor seco.

\section{SUMMARY}

The present research core-experimental type was developed with the purpose of determining the antihypertensive effect of the hydroalcoholic extract of the leaves of Xanthium catharticum HBK. "dry love" in rats with induced hipertension because L-NAME. Using samples of Xanthium catharticum HBK collected in the Mollepata town center, located in Ayacucho region. Secondary metabolites present in the extract were determined using coloration and precipitation specific tests. To determine antihypertensive effect. It was used the induction method by chronic administration with L-NAME in 30 rats, randomly divided into six groups, the first one received distilled water; the second one, L-NAME $40 \mathrm{mg} / \mathrm{kg}$; the third, fourth and fifth groups received L-NAME $40 \mathrm{mg} / \mathrm{kg}$ plus $100,200,400 \mathrm{mg} / \mathrm{kg}$ of extract, respectively; the sixth group received L-NAME $40 \mathrm{mg} / \mathrm{kg}$ plus captopril $100 \mathrm{mg} / \mathrm{kg}$. Data were subjected to analysis of variance (ANOVA) with $95 \%$ confidence. The results indicated the presence of sesquiterpenes, alkaloids, phenolics, flavonoids, saponins, amino acids and anthocyanins compounds. The highest percentage of dose antihypertensive efficacy was $400 \mathrm{mg} / \mathrm{kg} 40,58 \%$, while the groups of 100 and 200 $\mathrm{mg} / \mathrm{kg}$, got $33,96 \%$ and $28,54 \%$, respectively, with a mildly hypertensive behavior $(p<0,05)$ compared to control group receiving captopril 100 $\mathrm{mg} / \mathrm{kg}$ with a decrease of $99,40 \%$. It was conclude, that under experimental conditions, the hydroalcoholic extract of the leaves of Xanthium catharticum HBK "dry love" shown to have moderate antihypertensive effect.

Keywords: Hypertension, Xanthium catharticum HBK, L-NAME, dry love.

\section{INTRODUCCIÓN}

a enfermedad cardiovascular se ha convertido en una epidemia no transmisible que genera un preocupante alto costo directo e indirecto. Actualmente, se sabe que diversos factores, como herencia, edad, raza, tabaco, falta de ejercicio, obesidad, estrés crónico y exceso de sal en la dieta (1), pueden conllevar a sufrir de hipertensión arterial, una enfermedad crónica que conlleva entre 20 a $50 \%$ de las defunciones a nivel mundial ${ }^{(2)}$. La hipertensión arterial es un problema único en la terapéutica y la necesidad de estudiar su posible tratamiento ha llevado a desarrollar diversos procedimientos para producirla de forma sostenida en animales de experimentación; uno de los métodos más usados en la actualidad es la administración de L-NAME ( N $\omega$-nitro-L-arginina metil éster) ${ }^{(3)}$.

A pesar del gran desarrollo alcanzado por la síntesis química, en la actualidad las plantas medicinales continúan siendo un valioso arsenal de sustancias, o de precursores de las mismas, ya sea en forma de medicamento vegetal o de materia prima para la industria farmacéutica. Muchas de estas plantas utilizadas popularmente con fines medicinales, no cuentan con estudios farmacológicos que validen las actividades terapéuticas atribuidas que se les atribuye, por lo que es importante realizar estudios para poder contribuir con la población que las usa, dando a conocer su potencial terapéutico para que puedan ser empleadas de forma adecuada y segura ${ }^{(4)}$.

Es conocida la existencia de plantas con efecto antihipertensivo, y aunque también se atribuye esta capacidad a Xanthium catharticum HBK "amor seco", planta nativa de Sudamérica ${ }^{(5)}$, no existen al presente estudios al respecto, por lo que se propuso realizar la presente investigación, para lo cual se planteó el objetivo de evaluar la actividad antihipertensiva del extracto hidroalcohólico de las hojas de Xanthium catharticum HBK "amor seco". 


\section{MATERIAL Y MÉTODOS}

Material. Hojas de Xanthium catharticum HBK "amor seco", que crecen en el centro poblado de Mollepata, ubicado en la provincia de Huamanga, región Ayacucho a 2761 metros de altitud. Se recolectaron $300 \mathrm{~g}$ de hojas secas de Xanthium catharticum HBK "amor seco" durante el mes de agosto del 2012. Una parte de la planta fue llevada al Museo de Historia Natural de la Universidad Nacional San Cristobal de Huamanga (UNSCH) para la identificación taxonómica.

Se trabajó con 30 ratas, con pesos entre 150-200 g.

Diseño metodológico. Básico-experimental.

Preparación del extracto hidroalcohólico. Se uso la técnica de Miranda y Cuellar ${ }^{(6)}$. El material seco y pulverizado de las hojas (300 $\mathrm{mg}$ ) fue macerado con $1,5 \mathrm{~L}$ de solvente hidroalcohólico $80^{\circ}$ en un frasco ámbar por siete días a temperatura ambiente. Se filtró al vacío y el filtrado se concentró a sequedad en un rotavapor.

Tamizaje fitoquímico. Se realizaron las pruebas directamente en el extracto con reacciones específicas de coloración y precipitación, según Miranda y Cuellar ${ }^{(6)}$.

\section{Determinación del efecto antihipertensivo}

\section{Procedimiento experimental}

- Después de dos meses de aclimatación, se procedió a pesar los animales distribuyéndolos en seis grupos $(\mathrm{n}=5)$ para medir la presión arterial (PA) basal, denominándose al día en que se empezó con este procedimiento Do (día cero). Se empleó el medidor de PA Nib Panlab ${ }^{(7)}$.

- Enseguida se indujo hipertensión arterial en cinco grupos, mediante la administración de L-NAME en dosis de $40 \mathrm{mg} / \mathrm{kg} /$ día vía oral (v.o.) durante 10 días, según el método de Pereira et al., citado por Rojas ${ }^{(8)}$.

- El tratamiento con las sustancias de prueba (a dosis de $100,200,400 \mathrm{mg} / \mathrm{kg} /$ día) y captopril $100 \mathrm{mg} / \mathrm{kg} /$ día comenzó dos díasdespués de haber iniciado la administración de L-NAME D3 (día tres) y continuó hasta el Dio (día diez).

- La medición de la PA se realizó estrictamente a las o9 h 30 minutos, antes de aplicar cada tratamiento para evitar la variación circadiana.

- La presión arterial se siguió midiendo en los días $\mathrm{D}_{3}$, D4, D7 y Dio, respectivamente.

- Se registraron datos de presión arterial sistólica (PAS) y presión arterial diastólica (PAD). Los valores obtuvieron en mmHg.

- La presión media (PAM) se calculó mediante:

$$
\mathrm{PAM}=\mathrm{PAD}+\frac{1}{3}(\mathrm{PAS}-\mathrm{PAD})
$$

- El porcentaje de eficacia antihipertensiva (\%EAH) se calculó con la siguiente fórmula:

$$
\% \mathrm{EAH}=\frac{\mathrm{PA}_{\text {inicial }}-\mathrm{PA}_{\text {final }}}{\mathrm{PA}_{\text {inicial }}} \times 100
$$

\section{ANÁLISIS DE DATOS}

Los resultados fueron sometidos al análisis de varianza (ANOVA). Las diferencias intergrupos se analizaron por la prueba de Tukey, se uso un nivel de significancia $p<0,05$, software estadístico SPSS versión 17,0 y Microsoft Office Excel 2007.

\section{RESULTADOS}

Tabla 1. Metabolitos secundarios presentes en el extracto hidroalcohólico de las hojas de Xanthium catharticum HBK. "amor seco".

\begin{tabular}{lcc}
\hline \multicolumn{1}{c}{ Ensayo } & Metabolitos & Resultados \\
\hline Cloruro férrico & Taninos y fenoles & ++ \\
Espuma & Saponinas & + \\
Dragendorf & Alcaloide & +++ \\
Mayer & Alcaloide & +++ \\
Wagner & Alcaloide & ++ \\
Balyet & Lactonas & ++ \\
Lieberman- & Triterpenos y/o & ++ \\
Buchard & esteroides & ++ \\
Ninhidrina & Aminoácidos libres & + \\
Shinoda & Flavonoides & + \\
Antocianidinas & Antocianidinas & + \\
\hline
\end{tabular}

Excelente (+++) Buena (++) Escasa (+)

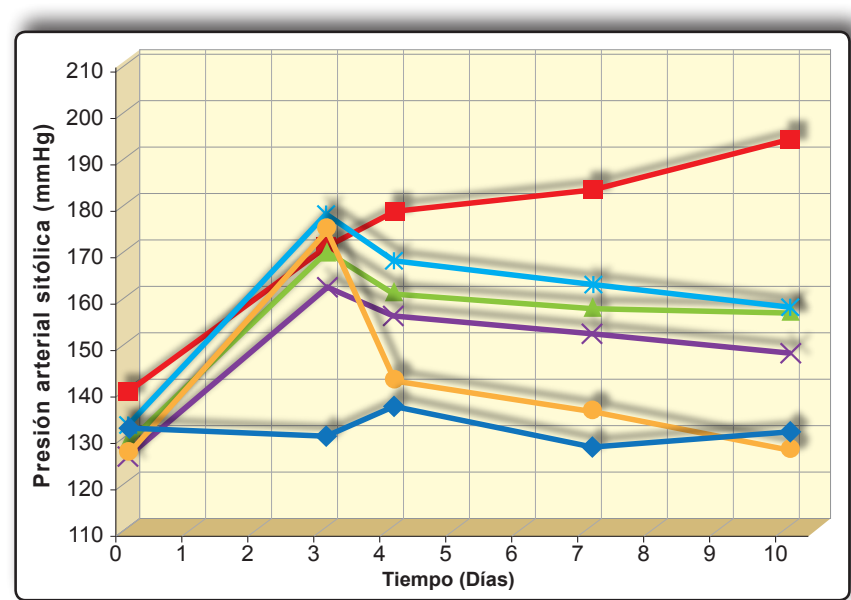

Figura 1. Variación de la presión arterial sistólica (mmHg) en función del tiempo por efecto del extracto hidroalcohólico.

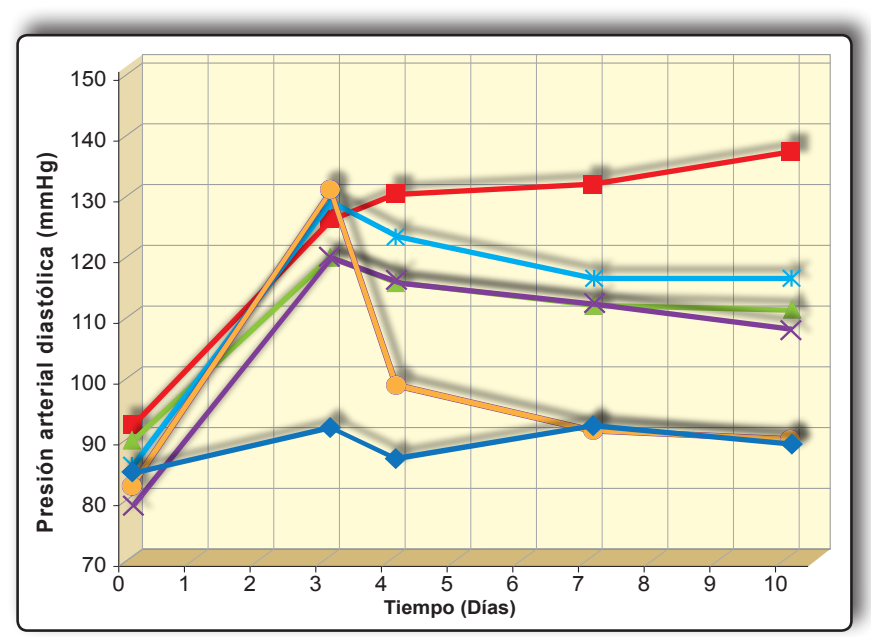

Figura 2. Variación de la presión arterial diastólica (mmHg) en función del tiempo por efecto del extracto hidroalcohólico. 
Tabla 2. Niveles de presión arterial de los grupos en los diferentes días de lectura.

\begin{tabular}{lccccc}
\hline Grupo & Día & PAS & PAD & PAM & \% EAH \\
\hline & D0 & $131,3 \pm 1,4$ & $83,9 \pm 1,5$ & $99,7 \pm 1,2$ & --- \\
Grupo I & D3 & $129,6 \pm 1,8$ & $91,3 \pm 0,5$ & $104,1 \pm 0,3$ & --- \\
H $_{2}$ (d) & D4 & $136,2 \pm 0,6$ & $86,3 \pm 0,9$ & $102,9 \pm 0,4$ & --- \\
& D7 & $127,6 \pm 1,5$ & $91,6 \pm 2,3$ & $103,6 \pm 1,1$ & --- \\
& D10 & $130,6 \pm 1,3$ & $88,6 \pm 1,4$ & $102,6 \pm 0,8$ & --- \\
Grupo II & D0 & $139,0 \pm 1,8$ & $91,6 \pm 2,1$ & $107,4 \pm 0,9$ & -- \\
L-NAME & $170,2 \pm 0,6$ & $120,5 \pm 0,9$ & $137,1 \pm 1,1$ & -- \\
40 my & D7 & $178,2 \pm 0,3$ & $125,6 \pm 0,6$ & $149,1 \pm 1,0$ & -- \\
& D10 & $182,5 \pm 1,1$ & $128,2 \pm 0,5$ & $146,3 \pm 0,5$ & -- \\
& D0 & $128,6 \pm 1,9$ & $132,5 \pm 0,7$ & $152,8 \pm 0,8$ & -- \\
Grupo III & D3 & $169,3 \pm 0,5$ & $119,3 \pm 2,6$ & $102,3 \pm 0,7$ & --- \\
Extracto & D4 & $160,3 \pm 1,6$ & $114,8 \pm 0,6$ & $135,9 \pm 1,4$ & --- \\
100mg & D7 & $157,2 \pm 0,3$ & $111,2 \pm 0,6$ & $126,5 \pm 2,1$ & 5,4 \\
& D10 & $156,2 \pm 1,2$ & $110,6 \pm 0,3$ & $125,8 \pm 0,3$ & 7,1 \\
& D0 & $125,3 \pm 1,3$ & $78,3 \pm 3,0$ & $93,7 \pm 0,9$ & --- \\
Grupo IV & D3 & $161,9 \pm 0,4$ & $119,2 \pm 0,2$ & $133,4 \pm 1,6$ & --- \\
Extracto & D4 & $155,2 \pm 0,3$ & $115,3 \pm 0,6$ & $128,7 \pm 0,2$ & 4,1 \\
200mg & D7 & $151,6 \pm 0,9$ & $111,6 \pm 0,8$ & $124,9 \pm 0,5$ & 6,4 \\
& D10 & $147,3 \pm 0,3$ & $107,3 \pm 0,6$ & $120,6 \pm 1,6$ & 9,2 \\
Grupo V & D0 & $135,3 \pm 0,4$ & $85,2 \pm 4,0$ & $101,9 \pm 1,9$ & --- \\
Extracto & D3 & $176,9 \pm 0,3$ & $128,3 \pm 2,4$ & $144,5 \pm 0,1$ & --- \\
400mg & D4 & $167,5 \pm 0,6$ & $122,6 \pm 2,7$ & $137,6 \pm 0,8$ & 5,3 \\
& D7 & $162,4 \pm 0,9$ & $118,7 \pm 2,1$ & $133,3 \pm 2,1$ & 8,2 \\
& D10 & $157,3 \pm 1,6$ & $115,6 \pm 0,9$ & $129,5 \pm 1,3$ & 11,1 \\
& D0 & $126,3 \pm 2,4$ & $81,7 \pm 5,1$ & $96,6 \pm 5,3$ & --- \\
Grupo VI & D3 & $174,3 \pm 2,6$ & $130,2 \pm 0,3$ & $144,9 \pm 1,0$ & --- \\
Captopril & D4 & $141,8 \pm 0,2$ & $98,2 \pm 0,5$ & $112,7 \pm 2,6$ & 18,7 \\
& D7 & $135,2 \pm 0,5$ & $90,6 \pm 0,9$ & $105,5 \pm 2,3$ & 22,5 \\
& D10 & $127,2 \pm 0,5$ & $89,3 \pm 0,7$ & $101,9 \pm 0,3$ & 27,1 \\
\hline & & & & & \\
\hline
\end{tabular}

\section{DISCUSIÓN}

La hipertensión arterial experimental en el presente estudio fue inducida utilizando el método de Pereira et al., citado por Rojas ${ }^{(7)}$, método que usa como inductor al L-NAME (N $\omega$-nitro-L-arginina metil éster).

A nivel renal, L-NAME elimina la influencia natriurética del óxido nítrico, contribuyendoa la retención de sodio con elevación de la presión sanguínea, además se reduce la tasa de filtración glomerular y se registra un aumento de la actividad de renina plasmática ${ }^{(14)}$.

Al inhibirse la producción de óxido nítrico en los animales por administración de L-NAME, se hizo evidente su papel hipertensor a partir del día $3\left(\mathrm{D}_{3}\right)$, registrándose elevación de 139,0 $\pm 1,8 \mathrm{mmHg}$ (Do) hasta 170,2 $\pm 0,6$ $\mathrm{mmHg}$ (tabla 2) en la presión arterial sistólica, resultados concordantes con los de Rojas ${ }^{(7)}$, quien encontró una mayor elevación de la presión arterial en el tercer día del experimento desde 129,7 $\pm 1,4 \mathrm{mmHg}$ (Do) hasta 162,7 \pm 1,8 mmHg $\left(\mathrm{D}_{3}\right)$ de presión arterial diastólica, corroborado también por la literatura, donde se citan valores de presión arterial sistólica para ratas normotensas con valores que van desde 98 a $184 \mathrm{mmHg}{ }^{(15)}$. Estas referencias permiten afirmar que los valores obtenidos de_131,06 \pm 1,62 $\mathrm{mmHg}$ de presión arterial sistólica para ratas al inicio del presente estudio, se encuentran dentro del rango descrito como normal para la especie. Las similitudes y diferencias entre los valores obtenidos y reportados por

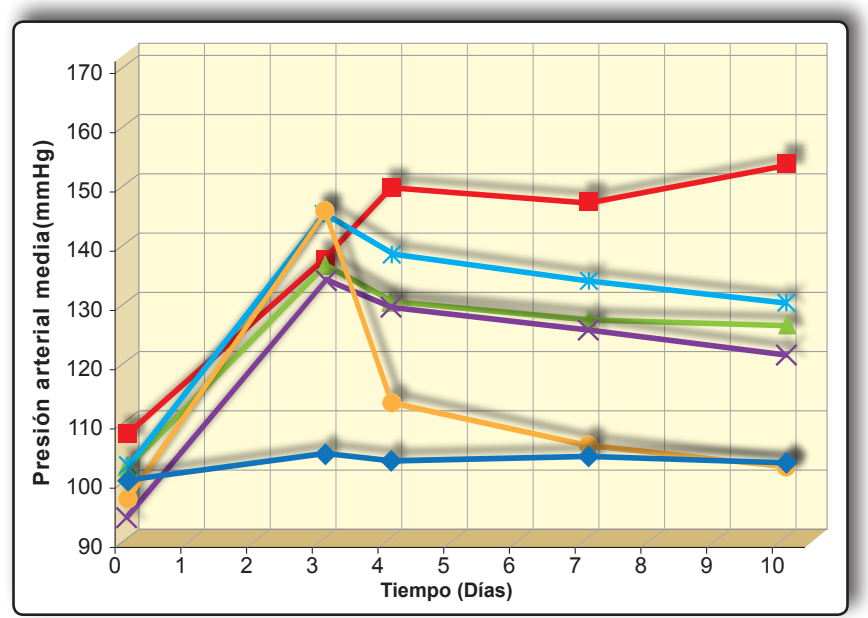

Figura 3. Variación de la presión arterial media (mmHg) en función del tiempo por efecto del extracto hidroalcohólico.

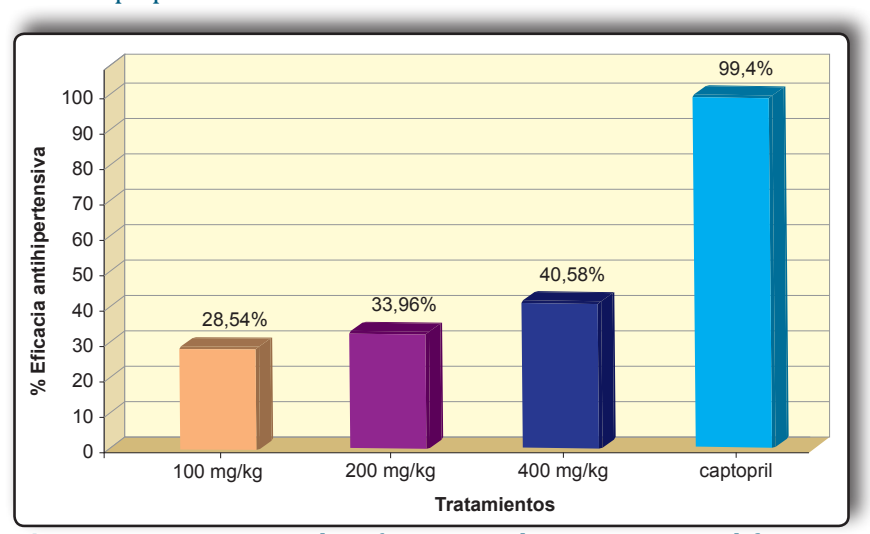

Figura 4. Porcentaje de eficacia antihipertensiva a diferentes concentraciones del extracto hidroalcohólico.

otros investigadores dependen de diversos factores como la susceptibilidad de la especie, el modo de exposición, la alimentación y el ambiente de trabajo.

En la figura 1, se observa la variación de la presión arterial sistólica en función del tiempo por efecto del tratamiento con los extractos de Xanthium catharticum HBK "Amor seco" a diferentes concentraciones y el estándar captopril.

La figura 4, muestra el porcentaje de eficacia antihipertensiva a partir del efecto generado sobre presión arterial sistólica en ratas hipertensas inducidas con L-NAME, producto de la administración de los extractos hidroalcohólicos de Xanthium catharticum HBK "amor seco" a diferentes concentraciones, encontrando un mayor porcentaje de eficacia $(40,58 \%)$ con $400 \mathrm{mg} / \mathrm{kg}$,; seguido por la dosis $200 \mathrm{mg} / \mathrm{kg}$ con $33,96 \%$ y por último $100 \mathrm{mg} / \mathrm{kg}$ con $28,54 \%$. Esto demuestra que los resultados obtenidos en la presión arterial sistólica son dosis dependiente.

Captopril,elfármacodereferencia, disminuyólapresión arterial de manera más efectiva que Xanthium catharticum HBK "amor seco" con 99,4\% de eficacia antihipertensiva, lo que confirma la eficiencia del tratamiento con inhibidores de la enzima convertidora de angiotensina I para prevenir la hipertensión en ratas con deficiencia de óxido nítrico. 
Es probable que el efecto antihipertensivo de Xanthium catharticum HBK "amor seco", demostrado en este estudio a nivel experimental, se deba a las acciones combinadas de los diferentes constituyentes químicos presentes en sus hojas.

El estudio fitoquímico preliminar realizado al extracto hidroalcohólico de las hojas de Xanthium catharticum HBK "amor seco" (tabla 1) demuestra la presencia de alcaloides, flavonoides, sesquiterpenos, compuestos fenólicos, saponinas, aminoácidos libres y antocianidinas, corroborando así el trabajo realizado por Cumanda et al. ${ }^{(16)}$ sobre la especie, en el cual, reporta la presencia de sesquiterpenos. Sin embargo, la actividad que se trata de demostrar recaería sobre los flavonoides, antocianidinas, fenoles y taninos, quienes contribuirían a reducir la presión arterial con sus propiedades diuréticas antioxidantes y vasorrelajantes, por incremento de la producción de óxido nítrico o por inhibición de la enzima convertidora de angiotensina I.

Estudios previos de Ojeda et al. ${ }^{(17)}$, han demostrado que existen en la naturaleza sustancias que son capaces de inhibir la enzima convertidora de angiotensina I (ECA): taninos, proantocianidinas, flavonoides, xantonas, ácidos grasos, alcaloides y terpenoides. Así lo demostró el extracto hidroalcohólico de Hibiscus sabdariffa L., rica en antocianinas caracterizadas como delfinidina-3-Osambubiosido y cianidina-3-sambubiosido, compuestos que inhibieron la enzima convertidora de angiotensina I (ECA).

Variosinformesdemuestrantambiénquelosflavonoides apigenina, luteolina, kaempferol-3-O-galactopiranosido, luteolina-7-O-glucopiranósido, quercetina glucoronido, quercetina 3-O-(6-galoilo)-galactosido, y quercetina-3-Ocafeoilglucósido-1,2-ramanósido, tienen capacidad para inhibir la ECA ${ }^{(13)}$. Por otra parte, la inhibición de esta enzima por taninos, especialmente por procianidinas oligoméricas está bien establecida para apichatechin flavan-3-ol y su hexámero ${ }^{(17)}$.

En otro bioensayo, utilizando enzimas ligadas (ELISA), Barbosa et al. ${ }^{(18)}$. verificaron que las hojas y tallos de Euphorbia hirta inhibieron la ECA en 90\%.

Algunos autores sugieren que estos compuestos anulan la actividad de la enzima al competir con el sitio activo, ya que se sabe que la ECA es estructuralmente una metalopeptidasa de zinc y funcionalmente una ectoenzima unida a membrana ${ }^{(17)}$.

Un mecanismo por el cual estos compuestos disminuirían la presión arterial, especialmente los compuestos polifenólicos y flavonoides, podría darse a través del incremento de la producción de óxido nítrico; se ha evidenciado que los flavonoides del jugo de uvas púrpura incrementan la liberación de óxido nítrico derivado de plaquetas y disminuyen la producción de superóxido. Asimismo, se ha comunicado que el flavonoide luteolina incrementó la actividad del promotor de la óxido nítrico sintetasa (ONS) endotelial, con el consecuente incremento del óxido nítrico ${ }^{(7)}$.

El efecto vasoprotector de los flavonoides ha sido atribuído especialmente al pigmento antocianina que es un potente antioxidante $y$ un capturador excepcionalmente potente de radicales libres de oxígeno, los cuales tienen un rol crítico en la patogénesis de la enfermedad cardiovascular ${ }^{(18)}$.

Es necesario realizar futuros estudios que permitan conocer qué compuestos presentes en la planta son los que producen estos efectos, pues dependiendo del propósito al que se destine, se puede obtener un extracto cuya composición química contiene la mayor parte de los constituyentes químicos de la planta, o uno que contenga sólo constituyentes con una determinada naturaleza ${ }^{(19)}$.

Se requiere establecer definitivamente las características toxicológicas de la planta antes de iniciar los estudios clínicos limitados y controlados en humanos, para comprobar su efectividad como antihipertensivo y establecer los parámetros de uso, limitaciones y efectos colaterales.

Se concluye que en condiciones experimentales el extracto hidroalcohólico de las hojas de Xanthium catharticum HBK "amor seco" evidencia tener moderado efecto antihipertensivo.

\section{REFERENCIAS BIBLIOGRÁFICAS}

1. Gonzales YN. Consumo de antihipertensivos en las farmacias privadas del distrito de Breña noviembrefebrero 2004. [Tesis]. Tesis Universidad Nacional de San Cristóbal de Huamanga. Huamanga, 2004.

2. Organización Mundial de Salud. Serie de informes Técnicos 844. Ginebra, 1998.

3. Rojas A. Evaluación del efecto de extracto etanólico de Durvillaea antarctica, sobre la presión arterial en ratas con hipertensión inducida con L-NAME. [Tesis para la Licenciatura] Facultad de Ciencias Veterinarias, Instituto de Farmacología. Universidad Austral de Chile. Santiago, 2008.

4. Cáceres A. Plantas medicinales de Guatemala. Guatemala: Edit. Universitaria de la Universidad San Carlos; 1995.

5. Cornejo V. Las Plantas y sus utilidades. Departamento Académico de Ciencias Biológicas, Área de Botánica. Huamanga: Universidad Nacional de San Cristóbal de Huamanga; 1983.

6. Miranda M, Cuellar A. Manual de prácticas de laboratorio - Farmacognosia y Productos Naturales. La Habana: Instituto de Farmacia y Alimentos, Universidad de La Habana, 2000.

7. NIBP. A Dinstruments making science easier. NIBP controller owner guide. 2011.

8. Rojas JP. Estudio pre-clínico y clínico de la seguridad y actividad antihipertensiva de Passiflora edulis Sims (maracuyá). [Tesis doctoral]. Facultad de Farmacia y Bioquímica, Universidad Nacional Mayor de San Marcos. Lima; 2009.

9. Rojas J, Ronceros S, Palomino R, Salas M, Azañero R, Cruz $\mathrm{H}$, Rojas A. Efecto coadyuvante del extracto liofilizado de Passiflora edulis (maracuyá) en la reducción de la presión arterial en pacientes tratados con enalapril. Anales de la Facultad de Medicina [Internet]. 2009 [Citado 8 octubre 2012]; $70(2): 103-8$. Disponible en: http:// revistasinvestigacion.unmsm.edu.pe/index.php/anales/ article/view/957/78o 
10. Ganong W. Fisiología Médica. 20 a ed. Ciudad de México: Editorial El Manual Moderno; 2006.

11. Guyton C, Hall J. Tratado de Fisiología Médica. 10 $0^{\underline{a}}$ ed. Madrid: Mc Graw Hill Interamericana; 2001.

12. Rodrigo J, Alonso D, Fernández AP, Serrano J, López JC, Encinas JM. Nitric oxide: synthesis, neuroprotection and neurotoxicity". [Internet] Anales Sis San Navarra [Internet]. 2000. [Citado 16 marzo 2013]; 23(2). Disponible en: http://scholar.google.com.pe/scholar?q= $\mathrm{El}+\% \mathrm{C}_{3} \% \mathrm{~B}_{3} x i d o+\mathrm{n} \% \mathrm{C}_{3} \% \mathrm{ADtrico} \%_{3} \mathrm{~A}+\mathrm{s} \% \mathrm{C}_{3} \% \mathrm{ADntesis}$ $\% 2 \mathrm{C}+$ neuroprotecci $\% \mathrm{C}_{3} \% \mathrm{~B}_{3} \mathrm{n}+\mathrm{y}+$ neurotoxicidad $\& \mathrm{hl}=\mathrm{es}$ \&as_sdt $=0 \%{ }_{2} C_{5} \& a s \_v i s=$

13. Adam A, Raij L. Nitric oxide, angiotensin 11 axis in renal and cardiovascular injury. [En línea] American Journal of Nephrology 2000. 13:211-20. Disponible en: http://www. karger.com/Article/Abstract/92984. [acceso o2 abril de 2013]; Nephorol [revista en internet].

14. Ramírez J, Palacios M, Gutiérrez O. Estudio del efecto antihipertensivo de la Salvia scutellarioides en modelo de ratas hipertensas. Colombia Médica [Internet]. 2006. [Citado 22 agosto 2012]; 37(1): 53-6o. Disponible en: http://colombiamedica.univalle.edu.co/index.php/ comedica/article/view/412/1098)

15. Pantanalli M. Valoración del efecto antihipertensivo del extracto liofilizado de hojas de Stachytarpheta cayennensis, sobre ratas con hiperetensión inducida con L-NAME. [Tesis pregrado]. Facultad de Ciencias Veterinarias, Instituto de Farmacología, Universidad Austral de Chile. Santiago, 2003.

16. Cumanda J, Marinoni G, De Bernardi M, Vidari G, Finzi P. New. Sesquiterpenes from Xanthium catharticum HBK. Journal of Natural Products [Internet]. 2001 [Citado o9 octubre 2012]; 54(2):460-465. Disponible en: http:// pubs.acs.org/doi/abs/10.1021/np50074a 017; Universidad Austral de Chile.

17. Ojeda D, Jiménez E, Zamilpab A, Herrera A, Tortoriellob J, Alvareza L. Inhibition of angiotensin convertin enzyme (ACE) activity by the anthocyanins delphinidinand cyanidin-3-O-sambubiosides from Hibiscus sabdariffa. Journal of Ethnopharmacology [Internet]. 2010 [Citado 02 abril 2012]; 127(1): 7-10. Disponible en: http://www.sciencedirect.com/science/article/pii/ So378874109006382

18. Barbosa J, Martins V, Rabelo L, Moura M, Silva M, Cunha E, et al. Natural products inhibitors of the angiotensin converting enzyme (ACE). A review between 1980 200o. Revista Brasileira de Farmacognosia [Internet]. 2006 [Citado 02 abril 2013]. 16(3): 421-46. Disponible en: http://www.scielo.br/scielo.php?pid=So102695X2006000300021\&script=sci_arttext

19. Herrera MO. Efecto de alcaloides tipo tropano presentes en Schizanthus grahamii sobre la presión arterial, en ratas normotensas e hipertensas. [Tesis de pregrado] Facultad de Ciencias Veterinarias, Instituto de Farmacología, Universidad Austral de Chile. 2003.

Manuscrito recibido el: 09/06/2015

Aceptado para su publicación el: 02/11/2015

\section{Correspondencia:}

Nombre: Mario Carhuapoma Yance

Dirección: Jr. Puno 1002 - Lima o1

e-mail: mariocarhuapomayance@hotmail.com 